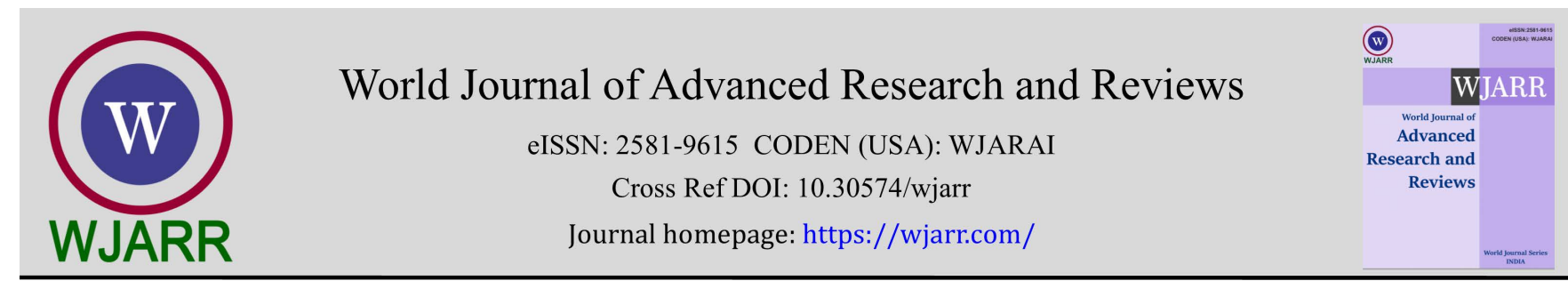

(REVIEW ARTICLE)

Check for updates

\title{
Unicystic ameloblastoma: Literature review
}

\author{
Luis Sebastián Pinos Pinos ${ }^{1,}{ }^{*}$, Jorge Antonio Reinoso Ortiz ${ }^{1}$, María Fernanda Torres Calle ${ }^{2}$ and Fernando \\ Mauricio Villalta Mendoza ${ }^{3}$
}

${ }^{1}$ Faculty of Dentistry, University of Cuenca, Cuenca, Ecuador.

${ }^{2}$ Associate Professor of Faculty of Dentistry, University of Cuenca, Cuenca, Ecuador.

${ }^{3}$ General Dentistry Faculty of Dentistry, University of Cuenca, Cuenca, Ecuador.

World Journal of Advanced Research and Reviews, 2021, 10(03), 095-103

Publication history: Received on 26 April 2021; revised on 04 June 2021; accepted on 07 June 2021

Article DOI: https://doi.org/10.30574/wjarr.2021.10.3.0240

\begin{abstract}
The Unicystic Ameloblastoma (UA), represents an invasive polymorphic lesion whose origin is currently unknown, but it is presumed to form from the epithelial remains of the Malassez from the epithelial sheath of Hertwig. It represents the second most prevalent odontogenic tumor, which is characterized by being locally invasive and of high recurrence. It usually presents a predilection for the male sex, affecting during the second and third decade of life. Clinically, it is asymptomatic, however, it can generate swelling with facial asymmetry, causing an expansion of the bone cortical, allowing infiltration into soft tissues. Radiographically, UA presents with a well-defined radiolucent unilocular appearance and histologically can be luminal, intraluminal and mural, depending on the characteristics of the pathological cavity. Treatment usually focuses on surgical resection of the lesion, which allows removal of the tumor with safe bone margins.
\end{abstract}

Keywords: Unicystic ameloblastoma; Diagnosis; Treatment; Prediction; Histology

\section{Introduction}

Ameloblastoma, according to the World Health Organization (O.M.S), is defined as "locally invasive polymorphic neoplasia that commonly has a follicular or plexiform pattern, in a fibrous stroma" [1-3]. It is not known exactly what the precursor stimulus of its development is, but it is believed to form from cellular remains from the enamel organ, dental lamina, or from epithelial remains of Malassez originating from the epithelial sheath of Hertwig [4]. The first time this pathology was described was in the study of Robinson and Martinez in 1977, defining it as unicentric, nonfunctional, intermittent growth, of a tumor nature, benign, clinically persistent and of conservative treatment $[5,6]$. The classification of ameloblastoma, according to the WHO in 2005, mentions four types, which are: solid or multicystic, unique, peripheral, and metastatic [7-10]. However, in the current classification provided by this organism in 2017 (Table.1), it is simplified into: conventional ameloblastoma, unicystic ameloblastoma, and the extraosseous/peripheral type [11]. The UA represents the second most common odontogenic tumor with a $10 \%$ prevalence, which affects exclusively the maxillae, presenting predisposition for the posterior region of the jaw. It is described with a benign behavior, but is characterized by being a locally invasive-destructive tumor, with a high rate of recurrence and has an incidence of 0.5 per million inhabitants per year, being more evident in countries from Africa and Asia, has a male predisposition and affects the second and third decade of life [4, 12-16].

The timely and correct diagnosis is of vital importance to obtain predictable and satisfactory results for the patient, therefore, the objective of this research is to carry out a review of the literature on UA, as well as providing information on the etiopathogenesis, its clinical, radiographic, histological characteristics, treatment and prognosis.

\footnotetext{
* Corresponding author: Luis Sebastián Pinos Pinos

Faculty of Dentistry, University of Cuenca.

Copyright (C) 2021 Author(s) retain the copyright of this article. This article is published under the terms of the Creative Commons Attribution Liscense 4.0.
} 
Table 1 WHO (2007) classification of Ameloblastoma variants

\begin{tabular}{|c|c|c|c|}
\hline \multicolumn{4}{|c|}{ Classification of Ameloblastomas according to histology } \\
\hline $\begin{array}{l}\text { Conventional } \\
\text { ameloblastoma }\end{array}$ & $\begin{array}{l}\text { Unicystic } \\
\text { ameloblastoma }\end{array}$ & $\begin{array}{l}\text { Ameloblastoma } \\
\text { Extra bone/ peripheral }\end{array}$ & $\begin{array}{l}\text { Ameloblastoma } \\
\text { Metastatic }\end{array}$ \\
\hline Acanthomatous & Luminal & \multirow{6}{*}{$\begin{array}{l}\text { Similar to conventional } \\
\text { ameloblastoma }\end{array}$} & \multirow{6}{*}{$\begin{array}{c}\text { Similar to conventional } \\
\text { ameloblastoma }\end{array}$} \\
\hline Basaloid & Intraluminal & & \\
\hline Desmoplastic & Mural & & \\
\hline Follicular & & & \\
\hline Granular cell & & & \\
\hline Plexiform & & & \\
\hline
\end{tabular}

\section{Material and methods}

A search of the literature of the last 20 years was carried out in the digital databases of "Pubmed", "Science direct", and "Scopus", using the following keywords: "unicystic ameloblastoma", "diagnosis", "prognosis" and "treatment", using the bolean connector " $A N D$ " and using the inclusion and exclusion criteria described below:

\subsection{Criteria for inclusion}

- Peer-reviewed articles from the last 20 years available in full text from digital databases: Pubmed, Science Direct, and Scopus.

- $\quad$ Literature reviews or systematic reviews on unicystic ameloblastoma.

\subsection{Exclusion criteria}

- Studies based on case reports, protocols, opinions, letters, and brief communications.

- $\quad$ Studies other than English.

Once the inclusion and exclusion criteria were applied, a total of 35 scientific articles were obtained.

\section{Results}

\subsection{Unicystic ameloblastoma (UA)}

The unicystic ameloblastoma (UA), a variant of ameloblastoma, represents 10 to $15 \%$ of the total intraosseous variants. The average age of onset is 25 years, where half of the cases affect the second decade of life [17], while other authors such as Harvey, P. et al., mentions an average UA diagnosis between 33 and 39 years of age; affecting about 10 per cent of children under 10 years of age [18]. According to Li, T.J. et al., there is greater predisposition in the second and third decade of life (70\%), especially at 25 years [16]. According to Ord, R.A. et al., it has a predominance at the age of 38.9 years [19]. It has a male predilection with a 1,6:1 ratio to female sex [17]. In Latin America, Ledesma-Montes, C. et al., report the incidence of this pathology in $22.7 \%$ of odontogenic tumors, with an average age of 26.3 years and affecting both sexes in the same way [20].

\subsection{Etiopathogenesis}

The etiology of ameloblastoma is still unknown, but based on the principles of a neoplasm, it can cause mutations or alterations in the genetic material of cells programmed for dental embryological development. Currently there is a tendency to investigate the circumstances that may modulate the incidence of this pathology, it has been shown that environmental and individual factors such as the state of general and nutritional health could play a role in the development of this disease [18]. This theory is based on the finding that the average age of onset of injury in industrialized countries is 10 to 15 years higher than that observed in developing countries. At the genetic level, a "BRAF" type activation mutation has been reported in the axon of chromosome 15, which results in the substitution of 
valine by glutamic acid on codon 600, taking the mutation name "BRAF600E". Research suggests that this biomarker is present in $63 \%$ in the UA, such mutant gene can be used as a biomarker to detect this pathology through an immunohistochemical technique $[2,11,21]$.

However, three mechanisms have been proposed for its pathogenesis:

- Basal cells of the reduced enamel epithelium associated with a developing tooth, remains of Malassez from the Hertwig lamina, heterotopic epithelials in extraoral sites; which undergo an ameloblastic transformation to give genesis to a unicystic cavity [2, 4, 10, 22, 23].

- It arises as a result of alteration of the epithelium of a Dentigerous Cyst (DC) or other type of odontogenic cyst, in which the neoplastic tissue of the ameloblastic epithelium is preceded by a non-neoplastic stratified squamous epithelial lining $[4,17,22]$.

- A conventional ameloblastoma undergoes a deformation of its islands with the following fusion of its multiple cysts giving rise to a cystic cavity [22].

Similarly, $50-80 \%$ of the cases of this pathology are related to an unborned tooth, mainly the third molar. $90 \%$ of the lesions are located in the posterior region of the jaw, followed by the parasymphysis and in the anterior and posterior areas of the jaw. Most of these lesions are related to DC in patients younger than 30 years; as well as: residual, root, primordial and globulomaxillary cysts $[2,4,10,11,17,18,22]$. Due to the similar clinical and radiographic characteristics, even in incisional biopsies the results may be inconsistent, it may also happen that the preoperative diagnosis of a dentifying cyst results in a unicystic ameloblastoma; after enucleation and subsequent histopathological analysis [21].

\subsection{Clinical characteristics}

This lesion is usually asymptomatic in early stages; however, large lesions generate painless swelling with facial asymmetry, caused by the expansion of the buccal or lingual cortical, allowing infiltration into soft tissues, being able to reach a size of $4.3 \mathrm{~cm}[14,17,18,24]$. Sometimes, the presence of pain is mentioned at the level of swelling, suppuration in the sinuses, nasal block, less frequent dental mobility, alteration in the dental eruption, malocclusion and poorly adjusted dental prostheses $[6,10,13,15]$.

\subsection{Radiographic features}

As an initial diagnosis, radiographic examinations including panoramic radiographs and computed tomography (CT) scans are necessary. Radiographically, UA appears with a well-defined radiolucent unilocular appearance (Figure 1).

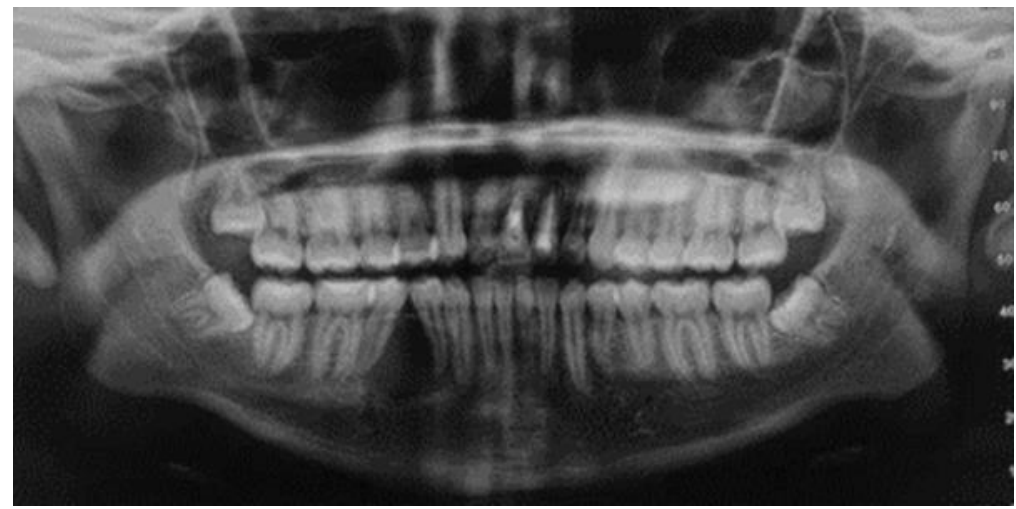

Image courtesy. Pineda-Alvarez D. Unicystic ameloblastoma panoramic X-ray [Universidad de Cuenca].2021 [Cited March 3, 2021]

Figure 1 Panoramic X-ray. Radiolucent lesion with defined edges, partially corticalized, in relation to dental parts 4.4 and 4.5 conditioning root divergence, extends from interdentary bone crest, towards mentonian hole

In the Zhang J study, it indicates that 43.3\% (92 out of 212 ameloblastomas) are unilocular, the UA are surrounded by a radiopaque halo, usually these lesions measure between 2 and $8 \mathrm{~mm}$ more than what is seen in the images [14], it is possible to observe a scalloping related to the crown of a third mandibular molar not erupted in 50 to $80 \%$ of cases (Figure 2). The UA can generate bone expansion (Figure 3), perforation of the cortical, as well as root resorption; in the radiological examination this may be confused with osteoblastomas or dentifying cysts $[1,5,10-12,14,15,17,22,23$, $25,26]$. When visualized in CT, it can be observed that there really are fenestrations that were evidenced in planar images [18]. 


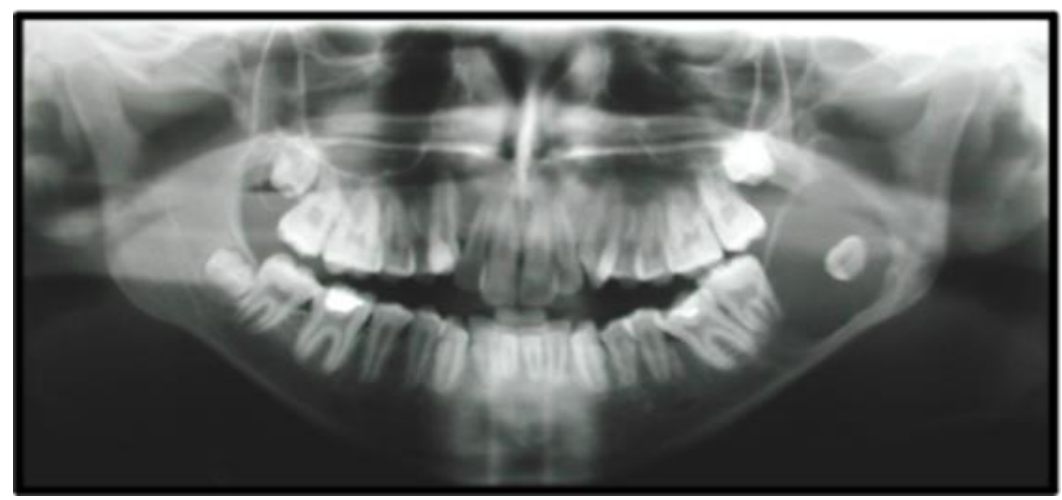

Image courtesy. Jaimes-Freyre N, Concha-Sánchez G. Unicystic ameloblastoma panoramic X-ray [Universidad Los Andes- Universidad de Chile].2012 [Cited March 3, 2021]

Figure 2 Panoramic X-ray. Radiolucent lesion, defined edges, partially corticalized, extends from mesial root of dental piece 3.7 to the middle area of ascending branch, generating expansion of the anterior wall, causing distal displacement of dental follicle of piece 3.8. Lesion projected immediately to the mandibular basal cortical, apparent caudal displacement of the inferior dentary duct

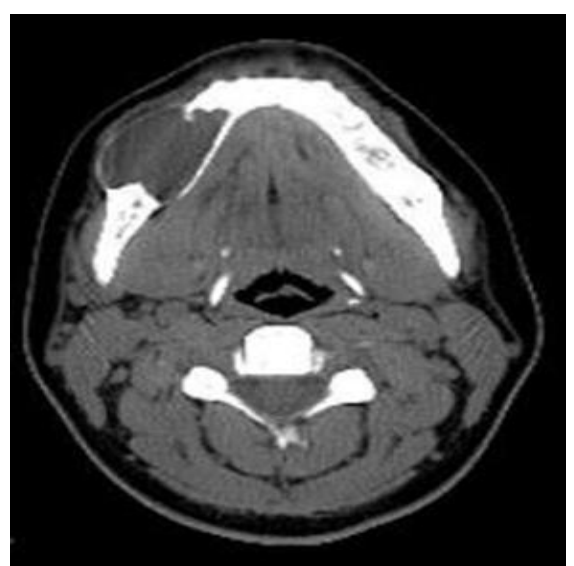

Image courtesy. Jaimes-Freyre N, Concha-Sánchez G. Unicystic ameloblastoma computerized tomography [Universidad Los Andes- Universidad de Chile].2012 [Cited March 3, 2021].

Figure 3 Computerized tomography. Radiolucent lesion with vestibular cortical expansion and thinning. In the axial section, hypodense lesion is observed in the mandibular body on the right side, with expansion of the bone board and marked thinning of vestibular cortical

\section{Histological characteristics}

Its name derives from its micro and macroscopic appearance. One of the main criteria for diagnosing UA is to find a unicystic space covered by an odontogenic epithelium [11,16, 27], the first classification was made by Robinson stating the following:

\subsection{Type I: Unique Luminal Lesion}

Unilocular cystic lesion with an ameloblastic epithelial lining that presents the criteria described by Vickers \& Gorlin [5, $10,13,28-30]$. The presence of columnar cells is described, hyperchromatic, with palisade nuclei and in inverse polarity; in addition, with subnuclear vacuoles located between the basement membrane and the nucleus. Finally, several cells form a thin overlay layer imitating the shape of a starry reticulum, and parakeratin layers are rarely seen (Figure.4, 5) $[18,22,27,31,32]$.

\subsection{Type II: Unique lesion plus intraluminal projections}

A nodule that arises from the cystic lining, which projects into the lumen or lumen of the cyst and has an odontogenic epithelium with a plexiform pattern [5, 10, 22, 28-31]. In addition, keratinization and an abrupt transition imitating the starry reticulum can be evidenced, plus hyperproliferation of the ameloblastic epithelium with lumen extension (Figure $4,5)[13,18,22,27]$. 


\subsection{Type III: Unique lesion plus intramural projections}

Cystic lesion with invasion of the epithelium to the connective tissue in follicular or plexiform form, the latter being the one that needs a much more aggressive treatment, it can also appear separated as islands (Figure 4, 5) $[5,10,13,22$, 27-31].

\subsection{Type IV: Unicystic lesion plus intramural and intraluminal projections}

It has characteristics of all the above (Figure 4, 5) [28, 31].

Ackermann currently proposes the following subgroups:

- Subtype 1: UA luminal.

- Subtype 1.2: UA luminal and intraluminal.

- $\quad$ Subtype 1.2.3: UA luminal, intraluminal, and intramural (Figure 4, 5).

- $\quad$ Subtype 1.3: UA luminal and intramural [22, 23, 27].

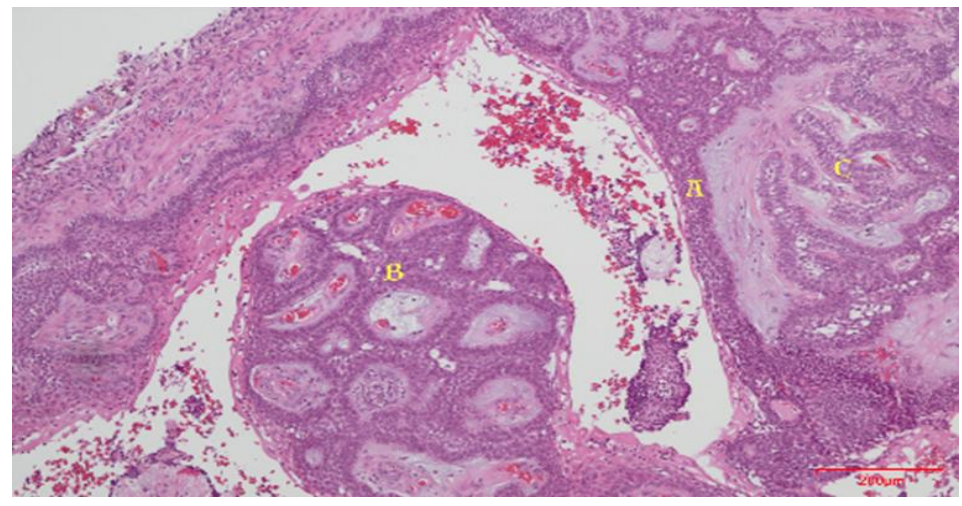

Figure 4 Microphotography showing UA luminal, intraluminal and mural. A. Luminal, B. Intraluminal, C. Mural. (H\&E 100x stain), Source: Own elaboration

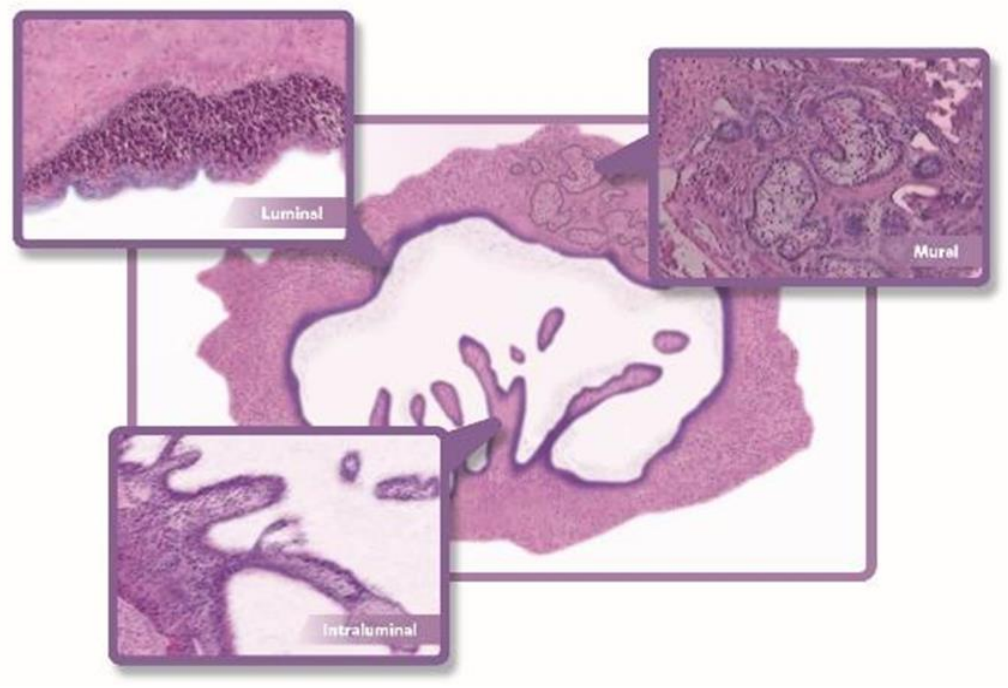

Figure 5 Image of the histological subtypes of UA described by Ackermann, Source: Own elaboration.

Ide F et al., indicates that when we have an initial lesion and we cannot detect the histological characteristics or patterns in it, it is recommended to make multiple cuts at different levels of the tumor [33]. For this reason, to avoid errors in the location and definition of UA histology, the entire wall should be evaluated histologically; however, this does not happen, in most cases one or two portions of the sample sent are analyzed, it is emphasized that in case of not reviewing the entire cystic lining, a diagnosis of mural variant can be allowed to pass, which has a higher rate of recurrence; in the same way when talking about an incisional biopsy the luminal or intraluminal variant can be found, leaving the mural affectation to pass; thus, UA excision biopsy is recommended [3, 33, 34] 


\section{Treatment and Recurrence}

Generally, the current treatment for ameloblastomas is the surgical path of resection, which consists of the removal of the block tumor with a wide bone margin and the delayed or immediate bone reconstruction of the defect with grafts or prosthetic rehabilitation; However, this "radical treatment" causes a high morbidity rate in the patient [11]. According to Hendra F.N et al., treatment of ameloblastoma depends on its location, size, extent, histopathological subtype, type of affected bone, and mandibular region [6]. According to Kim J. et al., when talking about the treatments accepted for UA, these can be: radical or conservative [31]. Another alternative treatment is marsupialization followed by enucleation. According to Li, T.J. et al., the relationship between the rate of recurrence and treatment is fundamental to the controversy that exists in defining the current approach for UA [16]. Therefore, Kim J. et al., mentions that one of the ways to evaluate the best treatment for a specific type of ameloblastoma is to assess the rate of recurrence; although the "unique ameloblastoma" is less aggressive than the solid type; this has a high recurrence (10\% to 25\%), many studies agree that the rate of recurrence of this tumor is lower when performing radical treatment [31]. Thus, a resection causes a recurrence of $3.6 \%$ and an enucleation recurrence in $30.5 \%$; then it is possible to conclude that eliminating an adequate bone margin is expected that the possibility of a recurrence is low, in addition, an adequate discernment is recommended when selecting the type of treatment in order to have maximum success. It is important to mention that radical surgery goes hand in hand with masticatory dysfunctions, abnormal movement of the jaw and removal of teeth; in addition in young patients the alteration in the mandibular growth could cause severe deformities, which may alter the quality of life of the patient [31]. In addition, the study by Hendra, F.N. et al., relates a comparison of radical treatment with conservative treatment, in which it is shown that the former has a lower rate of recurrence [8]. In the study by Pereira, N.B. et al., it is mentioned that the differential diagnosis between neoplasms and odontogenic cysts is fundamental, it is indicated that the conservative marsupialization of cysts is not an alternative for UA [21]. There is an exceptional case, which is the differential diagnosis between DC and UA; where neoplasia is enucleated with the provisional preoperative clinical diagnosis of DC, this is because both pathologies have radiographic similarities, clinical, and incisional biopsy may not have characteristics consistent with the definitive diagnosis [21]. According to Giraddi, G.B et al., the treatment for the "mural ameloblastoma" should be a resection with a safe margin of $1 \mathrm{~cm}$; compared to intraluminal and luminal variants, where the treatment of choice proposed is an enucleation with peripheral osteotomy followed by the application of Carnoy solution [6]. In addition, it is emphasized that the first-choice treatment in oral and maxillofacial tumors in children should be conservative and minimally invasive [6]. Whereas, in the study of Progel M.A. et al., an enucleation is recommended for the intraluminal subtype but not for the mural subtype; however, since these cannot be identified preoperatively, a more aggressive treatment with an osteotomy could be chosen or as an alternative enucleation is performed in conjunction with a subsequent treatment of the surrounding bone, where liquid nitrogen, Carnoy solution or a similar physico-chemical form is applied [30].

According to Parmar S. et al., the choice of treatment is facilitated by taking as a reference the subtypes of UA; where type I and II lesions can be treated conservatively by means of simple enucleation; type III and IV need more invasive and aggressive treatments, unfortunately the definitive diagnosis is obtained after the surgical process and with its subsequent evaluation [10].

The data obtained on recurrence indicate that there is a strong influence and direct relationship with the surgical procedure performed, a rate has been seen ranging from $10.7 \%$ to $25 \%$, finding less recurrence when comparing with the "conventional ameloblastoma" [18].

The data indicate a recurrence of $3.6 \%$ for resection, $30.5 \%$ for enucleation only, $16 \%$ for enucleation, followed by application of the Carnoy solution and $18 \%$ for marsupialization [22, 34, 35]. The site of greatest recurrence is the jaw in 80\%; preferably in the mandibular angle or gonion, there is a relationship of 3 to 1 linked to antero mandibular zones, here it is noted that this recurrence is recorded according to racial groups, where Asians show a lower predilection for injuries at the mandibular angle level compared to whites and blacks, while black people show a higher frequency at the mandibular antero part [18]. In children and adolescents the conservative treatment is recommended because at this time most of the lesions are unique, it was shown that the recurrence in these cases is scarce [10].

\section{Prognosis}

UA biologically has a less aggressive behavior, also has a good prognosis, even after conservative surgical treatment [5]. Adeyemo, W.L. et al., describes that the prognostic-treatment relationship is more relevant than the prognostichistological type relationship [24], on the other hand, Antonoglou, G. N. et al., indicates an important relationship between the histological type and the prognosis of UA [9]. Pogrel, M. A. et al., says that a more aggressive treatment than curettage and enucleation would significantly improve the prognosis of UA [30]. The "unicystic ameloblastoma" has a 
good prognosis [19], however, the literature relates that luminal UA represent a less aggressive type of ameloblastoma, so this variant has a better prognosis [13]. In addition, it is mentioned that the luminal and intraluminal subtypes owe their best prognosis to not having ameloblastomatous proliferation in the cyst wall [6].

\section{Conclusion}

The unicystic ameloblastoma represents one of the most frequent odontogenic tumors at the level of the maxillae, presenting a predilection in the male sex, by certain racial groups and is frequently found in the second and third decade of life. To date, it has not been possible to obtain a clear view of its origin; however, most theories are inclined to an epithelial mutation that forms a single cystic cavity. Its prognosis and recurrence are closely linked to its treatment and histological type; there is ambiguity in the "gold standard" of treatment and the form of diagnosis. More studies are therefore needed to establish a fixed guideline for its treatment.

\section{Compliance with ethical standards}

\section{Acknowledgments}

Acknowledgments to the dentists who allowed the preparation of the research.

\section{Disclosure of conflict of interest}

All the authors state no conflict of interest for publishing this original manuscript.

\section{References}

[1] Torres Lagares D, Infante Cossío P, Hernández Guisado JM, Gutiérrez Pérez JL. Ameloblastoma mandibular: Revisión de la literatura y presentación de seis casos \%J Medicina Oral, Patología Oral y Cirugía Bucal (Ed. impresa). 2005; 10: 231-8.

[2] González-González R, López-Verdín S, Lavalle-Carrasco J, Molina-Frechero N, Isiordia-Espinoza M, CarreónBurciaga RG, et al. Current concepts in ameloblastoma-targeted therapies in B-raf proto-oncogene serine/threonine kinase V600E mutation: Systematic review. World J Clin Oncol. 2020; 11(1): 31-42.

[3] Bansal S, Desai RS, Shirsat P, Prasad P, Karjodkar F, Andrade N. The occurrence and pattern of ameloblastoma in children and adolescents: an Indian institutional study of 41 years and review of the literature. International Journal of Oral and Maxillofacial Surgery. 2015; 44(6): 725-31.

[4] Morice A, Neiva C, Fabre M, Spina P, Jouenne F, Galliani E, et al. Conservative management is effective in unicystic ameloblastoma occurring from the neonatal period: A case report and a literature review. Oral surgery, oral medicine, oral pathology and oral radiology. 2020; 129(5): e234-e42.

[5] Hsu M-H, Chiang M-L, Chen J-K. Unicystic ameloblastoma. Journal of Dental Sciences. 2014; 9: 407-11.

[6] Giraddi GB, Arora K, Saifi AM. Ameloblastoma: A retrospective analysis of 31 cases. J Oral Biol Craniofac Res. 2017; 7(3): 206-11.

[7] Neagu D, Escuder-de la Torre O, Vázquez-Mahía I, Carral-Roura N, Rubín-Roger G, Penedo-Vázquez Á, et al. Surgical management of ameloblastoma. Review of literature. J Clin Exp Dent. 2019; 11(1): e70-e5.

[8] Hendra FN, Natsir Kalla DS, Van Cann EM, de Vet HCW, Helder MN, Forouzanfar T. Radical vs conservative treatment of intraosseous ameloblastoma: Systematic review and meta-analysis. Oral diseases. 2019; 25(7): 1683-96.

[9] Antonoglou GN, Sándor GK. Recurrence rates of intraosseous ameloblastomas of the jaws: a systematic review of conservative versus aggressive treatment approaches and meta-analysis of non-randomized studies. Journal of cranio-maxillo-facial surgery: official publication of the European Association for Cranio-Maxillo-Facial Surgery. 2015; 43(1): 149-57.

[10] Parmar S, Al-Qamachi L, Aga H. Ameloblastomas of the mandible and maxilla. Current opinion in otolaryngology \& head and neck surgery. 2016; 24(2): 148-54. 
[11] Shi HA, Ng CWB, Kwa CT, Sim QXC. Ameloblastoma: A succinct review of the classification, genetic understanding and novel molecular targeted therapies. The surgeon: journal of the Royal Colleges of Surgeons of Edinburgh and Ireland. 2020.

[12] Gomes CC, Duarte AP, Diniz MG, Gomez RS. Review article: Current concepts of ameloblastoma pathogenesis. Journal of oral pathology \& medicine: official publication of the International Association of Oral Pathologists and the American Academy of Oral Pathology. 2010; 39(8): 585-91.

[13] Seintou A, Martinelli-Kläy CP, Lombardi T. Unicystic ameloblastoma in children: systematic review of clinicopathological features and treatment outcomes. Int J Oral Maxillofac Surg. 2014; 43(4): 405-12.

[14] Mendenhall WM, Werning JW, Fernandes R, Malyapa RS, Mendenhall NP. Ameloblastoma. American journal of clinical oncology. 2007; 30(6): 645-8.

[15] Gordon SC, MacIntosh RB, Wesley RK. A review of osteoblastoma and case report of metachronous osteoblastoma and unicystic ameloblastoma. Oral Surg Oral Med Oral Pathol Oral Radiol Endod. 2001; 91(5): 570-5.

[16] Li TJ, Wu YT, Yu SF, Yu GY. Unicystic ameloblastoma: a clinicopathologic study of 33 Chinese patients. The American journal of surgical pathology. 2000; 24(10): 1385-92.

[17] Mortazavi H, Baharvand M. Jaw lesions associated with impacted tooth: A radiographic diagnostic guide. Imaging Sci Dent. 2016; 46(3): 147-57.

[18] Kessler H. Intraosseous ameloblastoma. Oral and maxillofacial surgery clinics of North America. 2004; 16: 30922.

[19] Ord RA, Blanchaert RH, Jr., Nikitakis NG, Sauk JJ. Ameloblastoma in children. Journal of Oral and Maxillofacial Surgery. 2002; 60(7): 762-70.

[20] Ledesma-Montes C, Mosqueda-Taylor A, Carlos-Bregni R, de León ER, Palma-Guzmán JM, Páez-Valencia C, et al. Ameloblastomas: a regional Latin-American multicentric study. Oral diseases. 2007; 13(3): 303-7.

[21] Pereira NB, Pereira KMA, Coura BP, Diniz MG, de Castro WH, Gomes CC, et al. BRAFV600E mutation in the diagnosis of unicystic ameloblastoma. 2016; 45(10): 780-5.

[22] Chaudhary Z, Sangwan V, Pal US, Sharma P. Unicystic ameloblastoma: A diagnostic dilemma. National journal of maxillofacial surgery. 2011; 2(1): 89-92.

[23] Singh A, Shaikh S, Samadi F, Saxena S, Verma R. Maxillary unicystic ameloblastoma: A review of the literature. National journal of maxillofacial surgery. 2011; 2: 163-8.

[24] Adeyemo WL, Bamgbose BO, Ladeinde AL, Ogunlewe MO. Surgical management of ameloblastomas: conservative or radical approach? A critical review of the literature. 2008; 1(1): 22-7.

[25] Rosenstein T, Pogrel MA, Smith R, Regezi J. Cystic ameloblastoma - Behavior and treatment of 21 cases. Journal of oral and maxillofacial surgery: official journal of the American Association of Oral and Maxillofacial Surgeons. 2001; 59: 1311-6.

[26] Zhang J, Gu Z, Jiang L, Zhao J, Tian M, Zhou J, et al. Ameloblastoma in children and adolescents. The British journal of oral \& maxillofacial surgery. 2010; 48(7): 549-54.

[27] Ricci M, Mangano F, Tonelli P, Barone A, Galletti C, Covani U. An unusual case of unicystic intramural ameloblastoma and review of the literature. Contemp Clin Dent. 2012; 3(Suppl 2): S233-S9.

[28] Dunsche A, Babendererde O, Lüttges J, Springer IN. Dentigerous cyst versus unicystic ameloblastoma--differential diagnosis in routine histology. Journal of oral pathology \& medicine : official publication of the International Association of Oral Pathologists and the American Academy of Oral Pathology. 2003; 32(8): 486-91.

[29] Lau SL, Samman N. Recurrence related to treatment modalities of unicystic ameloblastoma: a systematic review. Int J Oral Maxillofac Surg. 2006; 35(8): 681-90.

[30] Pogrel MA, Montes DM. Is there a role for enucleation in the management of ameloblastoma? Int J Oral Maxillofac Surg. 2009; 38(8): 807-12.

[31] Kim J, Nam E, Yoon S. Conservative management (marsupialization) of unicystic ameloblastoma: literature review and a case report. Maxillofac Plast Reconstr Surg. 2017; 39(1): 38.

[32] Motahhary P, Etebarian A, Asareh F. Granular cell type of a unicystic ameloblastoma: An unusual case and review of the literature. J Oral Maxillofac Pathol. 2014; 18(2): 331. 
[33] Ide F, Mishima K, Saito I, Kusama K. Diagnostically challenging epithelial odontogenic tumors: a selective review of 7 jawbone lesions. Head and neck pathology. 2009; 3(1): 18-26.

[34] Peacock ZS. Controversies in Oral and Maxillofacial Pathology. Oral Maxillofac Surg Clin North Am. 2017; 29(4): 475-86.

[35] Peacock ZS. Adjunctive Strategies for Benign Maxillofacial Pathology. Oral Maxillofac Surg Clin North Am. 2019; 31(4): 569-78. 